\title{
Population pharmacokinetics of cefazolin in maternal and umbilical cord plasma, and simulated exposure in term neonates
}

\author{
Omar Elkayal $\mathbb{D}^{1}{ }^{1}$, Karel Allegaert ${ }^{1,2,3}$, Isabel Spriet ${ }^{1,4}$, Anne Smits ${ }^{2,5}$, Marie-Christine Seghaye ${ }^{6}$, Corinne Charlier $^{7}$ \\ and Erwin Dreesen (D) ${ }^{1,8 *}$
}

\begin{abstract}
${ }^{1}$ Department of Pharmaceutical and Pharmacological Sciences, KU Leuven, Leuven, Belgium; ${ }^{2}$ Department of Development and Regeneration, KU Leuven, Leuven, Belgium; ${ }^{3}$ Department of Clinical Pharmacy, Erasmus MC Rotterdam, Rotterdam, The Netherlands; ${ }^{4}$ Pharmacy Department, University Hospitals Leuven, Leuven, Belgium; ${ }^{5}$ Neonatal Intensive Care Unit, University Hospitals Leuven, Leuven, Belgium; ${ }^{6}$ Department of Paediatrics, Centre Hospitalier Universitaire de Liège, Liège, Belgium; ${ }^{7}$ Department of Toxicology, Centre Hospitalier Universitaire de Liège, Liège, Belgium; ${ }^{8}$ Department of Pharmacy, Uppsala University, Uppsala, Sweden
\end{abstract}

*Corresponding author. E-mail: erwin.dreesen@kuleuven.be

Received 15 May 2021; accepted 14 August 2021

\begin{abstract}
Background: Intra-partum cefazolin is used to prevent group B Streptococcus (GBS) vertical transmission in mothers allergic to penicillin without a history of anaphylaxis.
\end{abstract}

Objectives: To investigate the maternal cefazolin dose-exposure relationship and subsequent maternal and neonatal target attainment at delivery.

\begin{abstract}
Methods: Data were obtained from 24 healthy, GBS-colonized pregnant women (20-41 years), undergoing vaginal delivery (gestational age $\geq 37$ weeks). During labour, all women received a $2 \mathrm{~g}$ cefazolin IV infusion. Eight hours later, eight women received another $1 \mathrm{~g}$ in the event of delayed $(>8 \mathrm{~h})$ delivery. Next to maternal plasma concentrations (up to 10 per dosing interval, until delivery), venous and arterial umbilical cord concentrations were determined at delivery. Target attainment in maternal/neonatal plasma was set at $1 \mathrm{mg} / \mathrm{L}$ for $60 \%$ of the dosing interval (unbound cefazolin, worst-case clinical breakpoint). A population pharmacokinetic (popPK) model was built (NONMEM 7.4). ClinicalTrials.gov Identifier: NCT01295606.

Results: At delivery, maternal blood and arterial umbilical cord unbound cefazolin concentrations were $>1 \mathrm{mg} / \mathrm{L}$ in 23/24 (95.8\%) and 11/12 (91.7\%), respectively. The popPK of cefazolin in pregnant women was described by a two-compartment model with first-order elimination. Two additional compartments described the venous and arterial umbilical cord concentration data. Cefazolin target attainment was adequate in the studied cohort, where delivery occurred no later than $6.5 \mathrm{~h}$ after either the first or the second dose. PopPK simulations showed adequate maternal and umbilical cord exposure for $12 \mathrm{~h}$ following the first dose.
\end{abstract}

Conclusions: PopPK simulations showed that standard pre-delivery maternal cefazolin dosing provided adequate target attainment up to the time of delivery.

\section{Introduction}

Group B Streptococcus (GBS) bacteria is one of the leading causes of maternal infections during pregnancy and post-partum, as well as in the neonate. ${ }^{1,2} \mathrm{GBS}$ is a colonizer of the gastrointestinal or genitourinary tract in about $20 \%$ of pregnant women. ${ }^{3}$ Vertical GBS transmission from the mother to the neonate occurs during labour or birth in $36 \%$ of colonized mothers, which may lead to meningitis, sepsis and other infections in the neonate. ${ }^{3}$ Therefore, antibiotic regimens are administered in mothers, to provide intra-partum GBS prophylaxis. The antimicrobial of choice for prophylaxis against GBS infections is penicillin $\mathrm{G}$ or amoxicillin. Both are deemed equally effective. ${ }^{4}$ However, for women that have penicillin allergy without a history of anaphylaxis, cefazolin is a safe and effective alternative in this setting. ${ }^{4,5}$

Cefazolin is a first-generation cephalosporin that works by inhibiting bacterial cell wall synthesis. It is only available for IV and intramuscular administration. Besides GBS prophylaxis, cefazolin is a standard prophylactic antimicrobial agent for various surgical interventions, including delivery-related interventions like caesarean section, owing to its broad-spectrum activity against most Gram-positive bacteria and some Gram-negative bacteria. ${ }^{6}$ It is highly bound to serum albumin (75\%-85\%), with unbound cefazolin available for pharmacological activity. ${ }^{7-11}$ The efficacy of cefazolin for GBS prophylaxis depends on the time that the unbound 
drug concentration is above the MIC, which is generally less than $1 \mathrm{mg} / \mathrm{L}$ for GBS, for $60 \%-70 \%$ of the dosing interval. ${ }^{12-15}$

Several population pharmacokinetic (popPK) models of cefazolin have been developed in different clinical settings. Two models described the PK of cefazolin during pregnancy. ${ }^{10,11}$ Three other models described the cefazolin PK during caesarean delivery. ${ }^{16-18}$ There are some data suggesting that labour influences the PK of antibiotics. ${ }^{19}$ However, no model described the PK of cefazolin during labour and subsequent vaginal delivery. Also, the subsequent post-delivery early neonatal exposure is unknown. Therefore, a popPK model will be of relevance, providing insight into the intra-partum neonatal protection against GBS infections.

The objectives of our work were: (i) to build a popPK model of cefazolin based on maternal and umbilical cord plasma concentrations obtained from women undergoing vaginal delivery; (ii) to investigate target attainment [time above $4 \times$ the epidemiological cut-off (ECOFF) of $0.25 \mathrm{mg} / \mathrm{L}$ ] in the mothers during labour; and (iii) to investigate the early post-partum cefazolin exposure in the newborn.

\section{Patients and methods}

\section{Patients and data collection}

Data were obtained from a prospective, exploratory study. The goal of the study was to obtain insight into target attainment and the PK of cefazolin in women undergoing vaginal delivery. Healthy singleton pregnant women were recruited. All women were colonized with GBS and were admitted for vaginal delivery. During labour, the women received a $2 \mathrm{~g}$ cefazolin IV infusion over $30 \mathrm{~min}$. If no delivery occurred $8 \mathrm{~h}$ after infusion, a second $1 \mathrm{~g}$ cefazolin infusion was given over 15 min. Maternal blood samples were obtained following a fixed sampling scheme with maximally 10 samples per dosing interval (until delivery), plus an additional sample right after delivery. Besides maternal blood samples, arterial and venous umbilical cord blood samples were collected at delivery. Additional data collected were gestational age, maternal age, maternal bodyweight and birthweight. The two parents' informed consents were collected according to the Ethics Committee approval of the Centre Hospitalier Universitaire de Liège before sample collection (ClinicalTrials.gov Identifier: NCT01295606).

\section{Analytical procedures}

All heparinized blood samples were centrifuged immediately after sampling (10 min, $1200 \mathbf{g}$, ambient temperature) and plasma was stored at $-80^{\circ} \mathrm{C}$ until further analysis. Cefazolin total plasma concentrations were measured using HPLC coupled with a diode array detector ('HPLC-DAD') with a lower limit of quantification of $0.5 \mathrm{mg} / \mathrm{L}$. The assay intra-day precision (expressed as relative standard deviation) varies from $0.9357 \%$ to $4.396 \%$ depending on concentration (repeatability), while the assay interday precision varies from $1.483 \%$ to $5.426 \%$ depending on concentration (intermediate precision). ${ }^{20}$

\section{PK/pharmacodynamic target}

An unbound cefazolin concentration above the MIC throughout at least $60 \%$ of the dosing period (i.e. $60 \% f T_{>\text {MIC }}$ ) was used as an optimal PK/pharmacodynamic target. ${ }^{21}$ In the absence of an MIC of cefazolin for GBS, we use the $0.25 \mathrm{mg} / \mathrm{L}$ ECOFF of amoxicillin for Streptococcus agalactiae. ${ }^{22,23}$ To avoid selecting resistant bacteria, a worst-case target of at least $60 \%$ $f T_{>4 \times E C O F F}$ was assumed.

Unbound cefazolin concentrations in maternal plasma were calculated from the measured total concentrations assuming $75 \%$ protein binding (lower limit of the $75 \%-85 \%$ interval, because albumin is known to decrease in the later stage of pregnancy). ${ }^{10,11}$

\section{PopPK modelling}

\section{Software}

Dataset formatting and exploration were performed using R (version 3.5.1, R Core Team, Vienna, Austria) using custom scripts based on packages including dplyr and ggplot2.24,25 PopPK analysis was performed using NONMEM (version 7.4; ICON Development Solutions, Gaithersburg, MD, USA), with a GNU Fortran 95 compiler and the Perl-speaks-NONMEM (PsN; version 4.7.0) toolkit on the interface software Pirana (version 2.9.7; Certara, Inc., Princeton, NJ, USA).

\section{Model development}

Parameter estimation was performed using first-order conditional estimation with interaction (FOCE-I) and differential equation solver ADVAN 13. The precision of the parameter estimates was evaluated based on the root squared error (RSE).

A base popPK model was developed. Different structural models with varying numbers of compartments were explored. Individual PK parameters were assumed to be log-normally distributed, which was achieved using an exponential function. Magnitudes of differences of individual parameters from the typical value (interindividual variability) and differences between observed values and model predicted values (residual variability) were quantified. The parametrization was performed using an exponential formula. For example, the clearance parametrization followed this equation:

$$
C L_{i}=T V C L \times \exp \left(\eta_{i}\right) \text { with } \eta_{i} \sim N\left(0, \omega^{2}\right)
$$

with $\mathrm{CL}_{i}$ the clearance of subject $\mathrm{i}$, TVCL the typical population value of the clearance, $\eta_{i}$ the individual subject's deviation from the typical value and $\omega^{2}$ the variance of inter-subject variability.

The base model was selected based on objective function value (OFV) comparisons (difference $\geq 3.84$ points; $P \leq 0.050$ ), Akaike information criterion (difference $\geq 3.84$ points; $P \leq 0.050$ ), plausibility and precision of parameter estimates, and goodness-of-fit plots. A final model, including covariate effects, was built via a two-way stepwise covariate modelling procedure (forward inclusion $\alpha=0.010$; backward elimination $\alpha=0.001$ ). The tested covariates were gestational age and maternal bodyweight.

\section{Final model evaluation}

The final model was evaluated using a prediction-corrected visual predictive check ( $p c V P C ; n=1000$ simulated replicates of the original dataset). Furthermore, bootstrapping was performed to obtain nonparametric estimates of uncertainty in parameter estimates $(n=2000$ bootstraps).

\section{Simulations in newborns}

Neonatal exposure after birth in our study cohort was predicted using Monte Carlo simulations. These simulations were based on the popPK model-predicted arterial umbilical cord concentrations at birth to enable simulations in all 24 cases. Subsequent cefazolin exposure in the newborns was predicted from the cefazolin popPK model of De Cock et al. ${ }^{26}$ The cefazolin PK was simulated 1000 times for each newborn. Missing neonatal serum albumin was multiply imputed $(n=1000)$ with values sampled from a uniform distribution within the range reported by Smits et al. ${ }^{8}(28.2-43.7 \mathrm{~g} / \mathrm{L})$. Postnatal age was set to zero and current weight was set equal to the registered birthweight in the current cohort of mother-newborn data. ${ }^{26}$ 


\section{Maternal and arterial umbilical cord simulations}

PK simulations were performed during standard pre-delivery maternal cefazolin dosing (see the Patients and data collection section). The maternal exposure and the PTA in arterial umbilical cord plasma were calculated up to $12 \mathrm{~h}$ after the first infusion. Arterial umbilical cord plasma concentrations were considered fetal plasma concentrations, since arterial cord blood originates directly from the fetal circulation. Based on previous literature, protein binding was assumed to be $75 \%$ in maternal plasma [see the PK/pharmacodynamic target section] and 60\% in neonatal plasma. ${ }^{10,11,26}$

\section{Results}

\section{Patient characteristics}

Data were obtained from 24 healthy pregnant women (20-41 years and bodyweight $70.5-89.7 \mathrm{~kg}$ ), colonized with GBS, undergoing vaginal delivery (gestational age $\geq 37$ weeks). A summary of participant characteristics is presented in Table 1. During labour, all women received a $2 \mathrm{~g}$ cefazolin IV infusion over $30 \mathrm{~min}$ for prophylaxis against GBS. In 16 women, delivery occurred within $6.5 \mathrm{~h}$ after the infusion [median $(\mathrm{IQR})=4.6(3.3-5.6) \mathrm{h}$ ]. Eight hours after the first administration, the remaining eight women received a second cefazolin infusion ( $1 \mathrm{~g}$ ) over $15 \mathrm{~min}$.

Altogether, the women contributed 245 plasma samples to the analysis, 24 of which were matched venous and arterial umbilical cord samples from 12 women (Figure 1). At the time of delivery, the maternal unbound cefazolin plasma concentrations were above the $1 \mathrm{mg} / \mathrm{L}$ target in 23/24 (95.8\%) women [5.82 (1.89-5.86) mg/L] (Figure S1, available as Supplementary data at JAC Online). The unbound $1 \mathrm{mg} / \mathrm{L}$ target was achieved in 11/12 (91.6\%) arterial umbilical cord samples [5.12 (3.56-5.72) mg/L].

\section{PopPK model}

\section{Model development}

A two-compartment popPK model with linear elimination best described the maternal cefazolin concentration data (Table S1). Two additional compartments were added to represent the venous and arterial umbilical cord concentrations, with blood flowing from the maternal central compartment to the venous umbilical cord compartment, then to the arterial umbilical cord compartment, and then back to the maternal central compartment with the placenta as a barrier between both (Figure 2).

Interindividual variability was estimated on the maternal clearance $[43 \%$ coefficient of variation (CV)], central volume of distribution $(67 \% \mathrm{CV})$ and peripheral volume of distribution $(68 \% \mathrm{CV})$. A mixed error model was selected to describe the residual variability based on goodness-of-fit plots and the OFV.

Gestational age and body weight were tested as covariates, but did not significantly improve the OFV or the parameter estimation accuracy. Therefore, these covariates were not retained in the final model (Table S2). Parameter estimates from the final model are shown in Table 2.

\section{Model evaluation}

Goodness-of-fit plots showed that the final model adequately described the observed data (Figure S2 and Figure S3). A PCVPC showed the good predictive capacity of our model, where the
Table 1. Summary of patient characteristics

\begin{tabular}{|c|c|}
\hline Parameter & Value \\
\hline \multicolumn{2}{|l|}{ Demographics } \\
\hline patients, $n$ & 24 \\
\hline age (years), median (IQR) & $30(26-32.3)$ \\
\hline gestational age (weeks), median (IQR) & $39(39-40)$ \\
\hline $\begin{array}{l}\text { maternal bodyweight at first cefazolin dose } \\
(\mathrm{kg}) \text {, median (IQR) }\end{array}$ & $80.5(70.5-89.7)$ \\
\hline birthweight (kg), median (IQR) & $3.5(2.7-4.6)$ \\
\hline \multicolumn{2}{|l|}{ Cefazolin dosing } \\
\hline cefazolin first dose (mg), median (IQR) & $2000(2000-2000)$ \\
\hline cefazolin second dose (mg), median (IQR) & $1000(1000-1000)$ \\
\hline number of cefazolin first doses, $n$ & 24 \\
\hline number of cefazolin second doses, $n$ & 8 \\
\hline \multicolumn{2}{|l|}{ Delivery } \\
\hline $\begin{array}{l}\text { time between first cefazolin dose and } \\
\text { delivery (h), median (IQR), } n \text { (cases) }\end{array}$ & $4.6(3.3-5.6), 16$ \\
\hline $\begin{array}{l}\text { time between second cefazolin dose and } \\
\text { delivery (h), median (IQR), } n \text { (cases) }\end{array}$ & $0.5(0.1-1), 8$ \\
\hline \multicolumn{2}{|l|}{ Sampling information } \\
\hline number of plasma samples, $n$ & 254 \\
\hline $\begin{array}{l}\text { number of plasma samples/patient, median } \\
\text { (IQR) }\end{array}$ & $10(9-11)$ \\
\hline $\begin{array}{l}\text { number of umbilical cord plasma samples, } \\
\text { n (arterial/venous) }\end{array}$ & $24(12 / 12)$ \\
\hline $\begin{array}{l}\text { number of plasma samples below the lower } \\
\text { limit of quantification, } n(\%)\end{array}$ & $0(0)$ \\
\hline \multicolumn{2}{|l|}{ Cefazolin measurements } \\
\hline $\begin{array}{l}\text { cefazolin maternal concentrations (mg/L), } \\
\text { median (IQR) }\end{array}$ & $61(23.3-99.4)$ \\
\hline $\begin{array}{l}\text { cefazolin arterial umbilical concentrations } \\
\text { (mg/L), median (IQR) }\end{array}$ & $12.8(8.9-14.3)$ \\
\hline $\begin{array}{l}\text { cefazolin venous umbilical concentrations } \\
\text { (mg/L), median (IQR) }\end{array}$ & $12.1(9-14.35)$ \\
\hline
\end{tabular}

median values and the $95 \%$ CIs of the prediction-corrected observed data fell within the $95 \%$ prediction intervals of the model (Figure 3). Median values of the non-parametric bootstrap were in good agreement with point estimates (Table 2).

\section{Simulations in newborns}

The PK simulations showed adequate neonatal antimicrobial exposure for protection against GBS in neonates at birth in our study cohort [3.58 (2.50-4.84) mg/L] $(n=24)$ (Figure 4). Unbound cefazolin is predicted to remain detectable for at least $24 \mathrm{~h}$ after birth.

\section{Maternal and arterial umbilical cord simulations}

The PK simulations showed that the median maternal unbound cefazolin plasma is above the therapeutic target of $1 \mathrm{mg} / \mathrm{L}$ for the entire $12 \mathrm{~h}$ simulation period (Figure $5 \mathrm{a}$ ). The PTA of neonatal (arterial) unbound cefazolin concentrations slowly increases and reaches $90 \%$ or more approximately $1 \mathrm{~h}$ after the start of infusion (Figure 5b). 


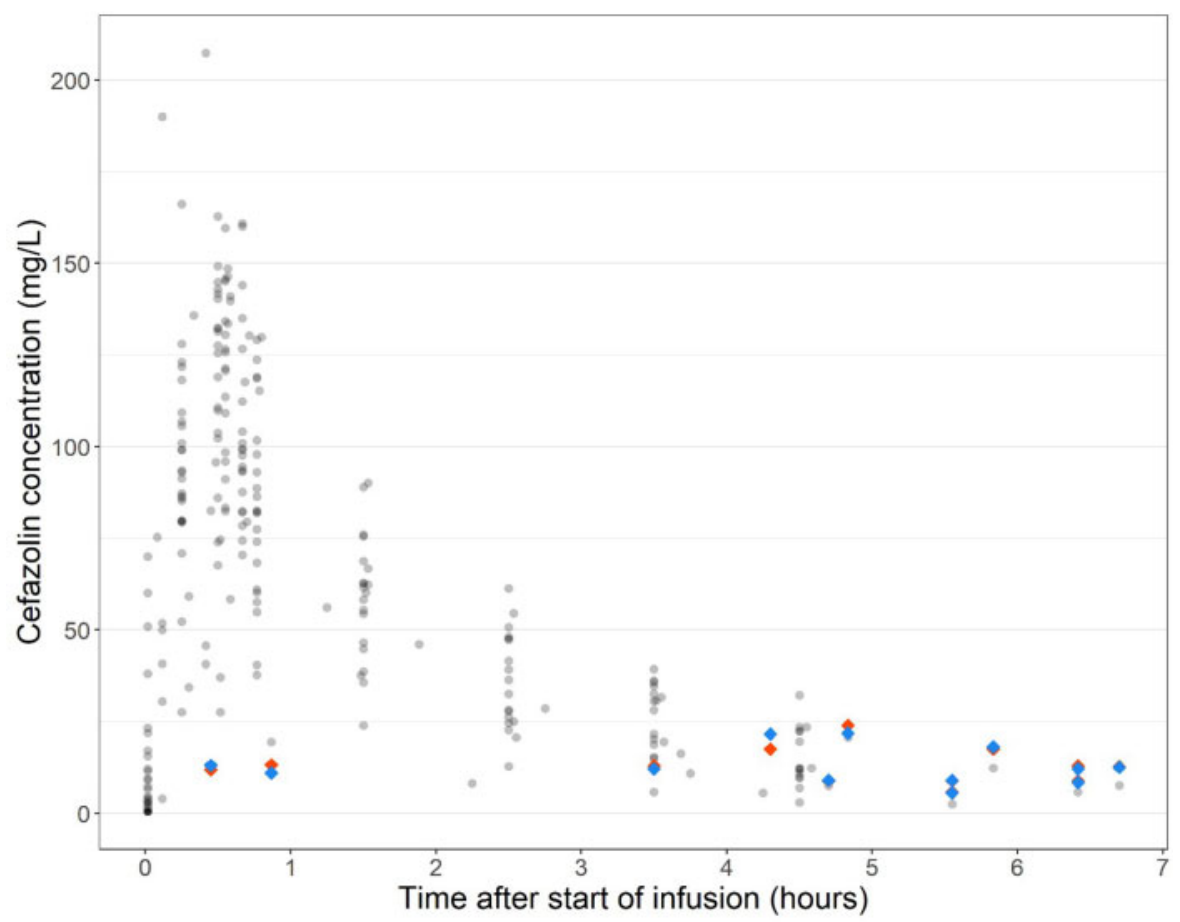

Figure 1. Total cefazolin concentration versus time after dose. Grey dots, maternal plasma concentrations; blue diamonds, venous umbilical cord plasma concentrations; red diamonds, arterial umbilical cord plasma concentrations. This figure appears in colour in the online version of JAC and in black and white in the print version of JAC.

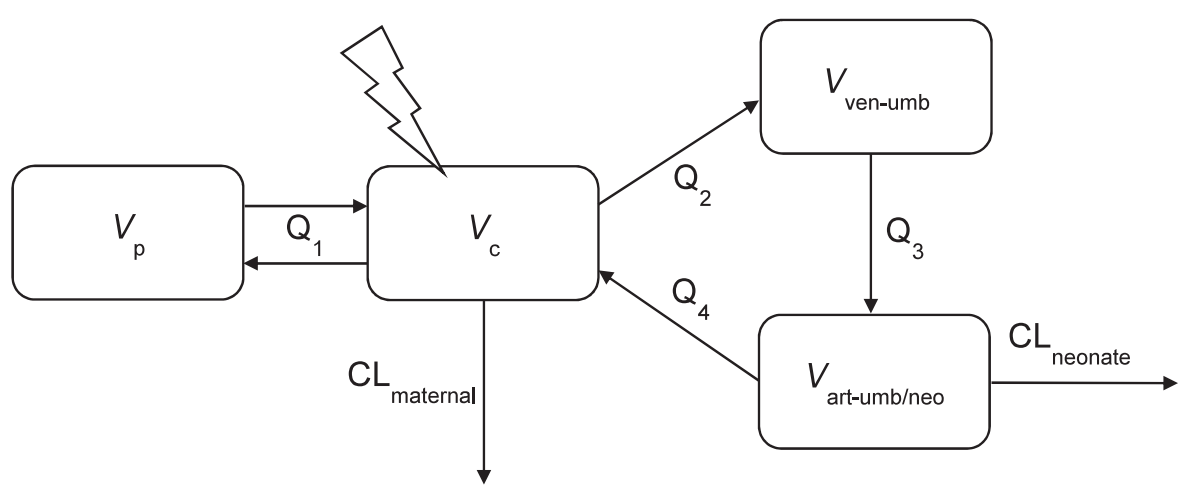

Figure 2. Schematic representation of the pre-delivery maternal-umbilical cord popPK model. art, arterial; $\mathrm{CL}$, clearance; neo, neonatal; Q, blood flow; umb, umbilical cord; $V$, volume of distribution; ven, venous.

\section{Discussion}

In this study, we performed a popPK modelling and simulation study of cefazolin in healthy women undergoing vaginal delivery and their newborns. We obtained a better understanding of the pre-delivery maternal cefazolin dose-exposure relationship and subsequent post-delivery duration of neonatal target attainment. Although PK variability is large, fixed $2 \mathrm{~g}$ cefazolin dosing-followed by $1 \mathrm{~g}$ cefazolin $8 \mathrm{~h}$ later if delivery had not occurred yetprovided adequate unbound cefazolin exposure in the mother and in the neonate at the time of birth.

A two-compartment model with linear elimination best described the maternal and umbilical cord concentration data. To the best of our knowledge, no popPK model of cefazolin in women in labour and subsequently undergoing vaginal delivery has been published before. However, there are two popPK models describing the cefazolin PK in women during pregnancy (gestational age range 17-40 weeks, fetal surgery or caesarean). ${ }^{10,11}$ Additionally, three popPK models are available describing cefazolin PK in women undergoing caesarean delivery. ${ }^{16-18}$ Two of the previous models (both caesarean delivery models) used a onecompartment model to describe the cefazolin popPK. ${ }^{16,17}$ This structural difference could be due to the sparse sampling schemes in these studies, as well as the physiological difference between vaginal and caesarean deliveries (different volume of distribution and clearance). In the previously published models, the most common covariates were maternal bodyweight, gestational age and maternal albumin. Bodyweight and gestational age were tested, 
Table 2. Population parameter estimates

\begin{tabular}{|c|c|c|}
\hline Parameter & Estimate (\%RSE) [\%shrinkage] & Bootstrap median $(95 \% \mathrm{CI}$ \\
\hline $\mathrm{CL}_{\text {maternal }}(\mathrm{L} / \mathrm{h})$ & $7.15(10)$ & $6.99(3.41-9.03)$ \\
\hline$Q_{1}(L / h)$ & $26.1(19)$ & $27.75(18.56-45.95)$ \\
\hline$V_{p}(L)$ & $6.95(23)$ & $7.05(4.04-9.71)$ \\
\hline $\mathrm{Q}_{2}(\mathrm{~L} / \mathrm{h})$ & $2.61(16)$ & $2.77(1.04-6.86)$ \\
\hline $\mathrm{Q}_{4}(\mathrm{~L} / \mathrm{h})$ & $5.47(14)$ & $5.49(2.27-10.91)$ \\
\hline $\mathrm{CL}_{\text {neonatal }}(\mathrm{L} / \mathrm{h})$ & O FIXED & O FIXED \\
\hline \multicolumn{3}{|l|}{ Interindividual variability model } \\
\hline on $\mathrm{CL}(\% \mathrm{CV})$ & $42.9(16)[10]$ & $45.8(26.3-80.8)$ \\
\hline on $V_{c}(\% C V)$ & $66.6(25)[8]$ & $65.3(17.9-114.1)$ \\
\hline on $V_{p}(\% C V)$ & $67.9(33)[26]$ & $71.8(22.5-158.3)$ \\
\hline additive residual variability (mg/L) (venous) & $0.021(58)[23]$ & $0.014\left(2.44 \times 10^{-6}-0.043\right)$ \\
\hline proportional residual variability (\%CV) (venous) & O FIX & O FIX \\
\hline additive residual variability (mg/L) (arterial) & O FIX & O FIX \\
\hline proportional residual variability (\%CV) (arterial) & $9.16(47)[5]$ & $8.21(0.43-17.81)$ \\
\hline
\end{tabular}

$C L$, clearance; $C V$, coefficient of variation calculated as $\sqrt{\exp \left(\omega^{2}\right)-1} \times 100 \%$; $V_{c}$, central volume of distribution; $V_{p}$, peripheral volume of distribution.

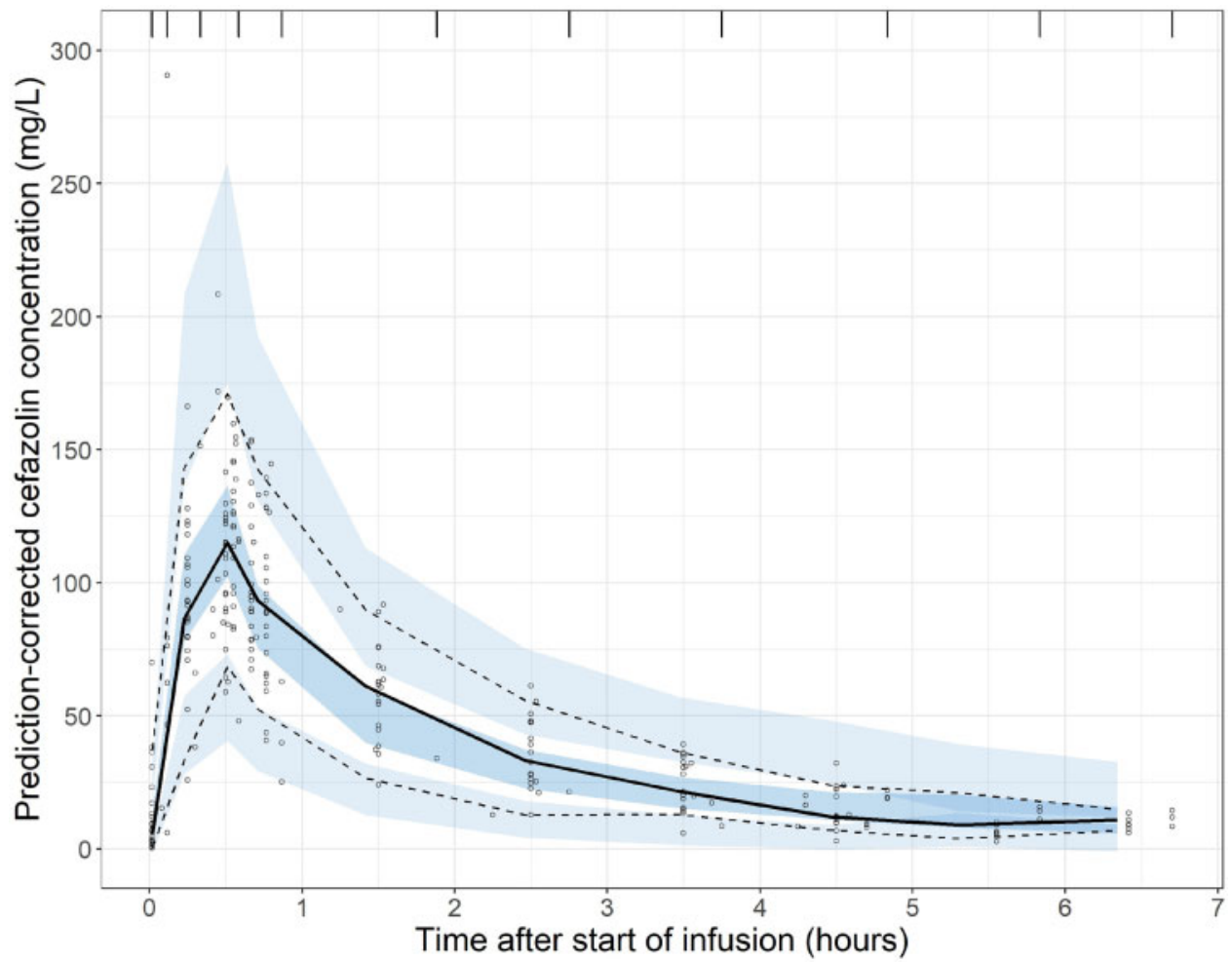

Figure 3. pcVPC of the final model. The observed prediction-corrected total cefazolin concentrations are represented by circles. The black continuous and broken lines represent the median and the 2.5th and 97.5th percentiles, respectively, of the prediction-corrected observations. The shaded areas indicate the $90 \%$ prediction intervals of the median and the 2.5 th and 97.5 th percentiles of the simulated values. This figure appears in colour in the online version of JAC and in black and white in the print version of JAC. 


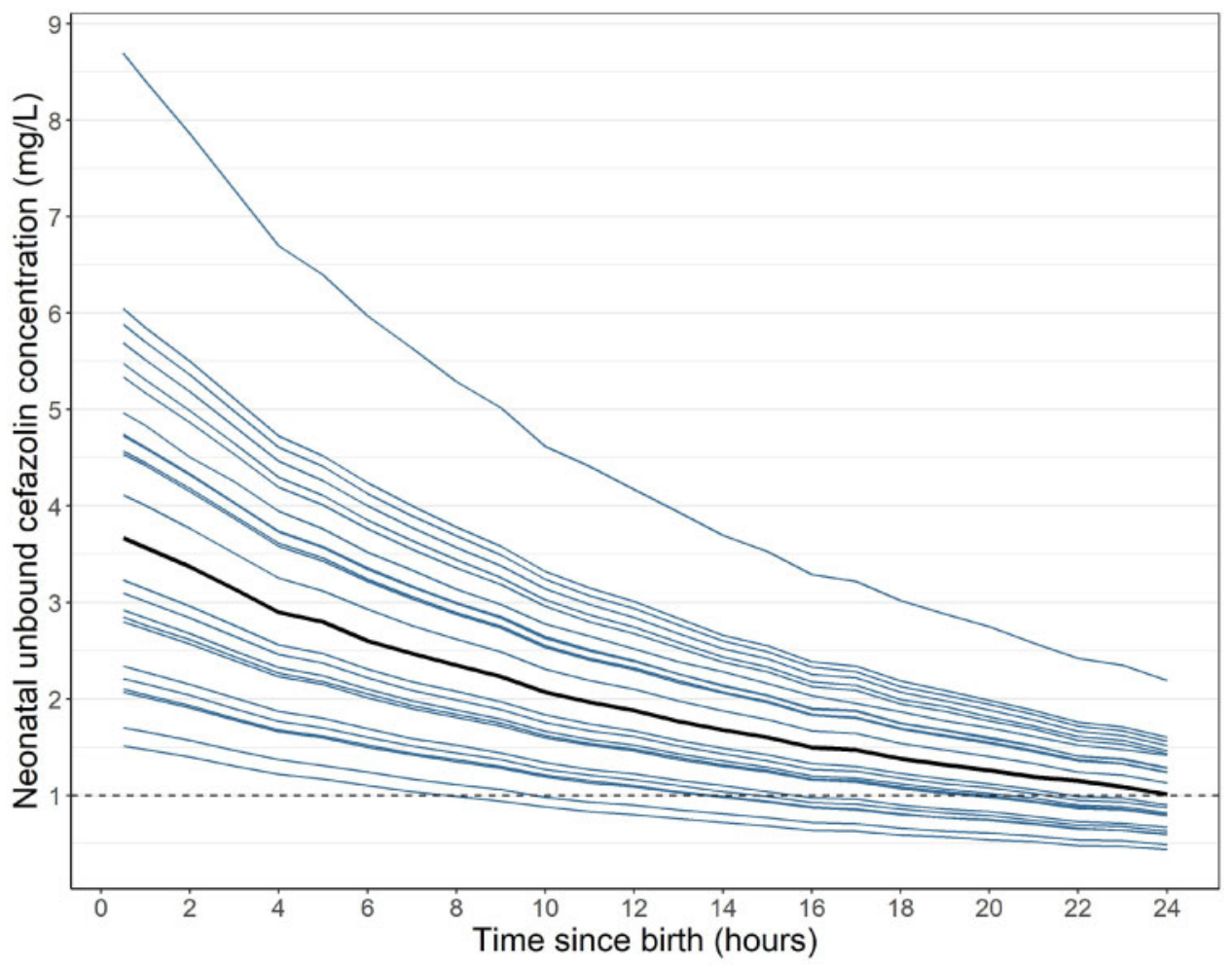

Figure 4. Neonatal unbound cefazolin concentrations versus time since birth. The blue lines each represent the median of 1000 simulations based on one of the predicted neonatal concentrations from the developed model. The thick black line represents the median trend. The horizontal broken line indicates the $1 \mathrm{mg} / \mathrm{L}$ therapeutic target. This figure appears in colour in the online version of JAC and in black and white in the print version of JAC.

but not retained in our model, while serum albumin concentrations were not available in our study dataset.

Muller et al. ${ }^{19}$ suggested that physiological changes during labour and subsequent vaginal delivery may influence the maternal PK of antibiotics (mainly distribution characteristics), as well as their placental transfer and subsequent fetal and neonatal exposure. Comparing our cefazolin PK parameters during labour with those reported by others during pregnancy and caesarean delivery, no clear differences could be observed. ${ }^{17,27}$ However, a future popPK model meta-based analysis is warranted to obtain further insight into potential differences.

Besides maternal PK, Muller et al. ${ }^{19}$ also indicated the need to document fetal and neonatal exposure. Our dataset included samples from the umbilical cord (venous and arterial) at the time of cord clamping. Unfortunately, no postnatal observations in the newborns of the mothers recruited were available in our study. However, similar to Muller et al., ${ }^{28}$ we postulated that the arterial umbilical cord concentrations reflect the neonatal concentrations at birth. Starting from these model-predicted arterial umbilical cord concentrations, subsequent neonatal exposure was simulated with the De Cock et al. ${ }^{26}$ model. Unbound cefazolin was predicted to remain above $0.5 \mathrm{mg} / \mathrm{L}$ for at least $24 \mathrm{~h}$ after birth. Consequently, a blood culture collected within this time frame, for example because of suspected early-onset sepsis in the newborn, is more likely to remain false negative.

Although target attainment was adequate in our study cohort, delivery occurred no later than $6.5 \mathrm{~h}$ after the first infusion and $4 \mathrm{~h}$ after the second infusion in all women. Therefore, we performed PK simulations, calculating the PTA at delivery across the $8 \mathrm{~h}$ after the first infusion and the $4 \mathrm{~h}$ after the second infusion (total $12 \mathrm{~h}$ period). These simulations showed that the unbound cefazolin concentration in the maternal plasma remains above the therapeutic target for the entire $12 \mathrm{~h}$ period of the simulations performed. Furthermore, the simulations also illustrated the slow increase in neonatal (arterial umbilical cord) unbound cefazolin concentrations, such that 90\% PTA is reached approximately $1 \mathrm{~h}$ after the start of the first infusion.

Our study had several strengths. First, our dataset included rich sampling, which enabled the accurate capture of the structural elements of the popPK model. Second, our data included umbilical cord samples, which enabled us to have insight into the neonatal target attainment. Our study had some limitations. First, we were limited with regard to covariate exploration, because our dataset had only 24 pregnant women, which limited the ability of our model to identify covariates. Additionally, we were limited with the covariates included in our dataset. Only two covariates were tested in the popPK model building (gestational age and maternal bodyweight) and both were not retained. In future research, it would be of interest to test other covariates, such as estimated glomerular filtration rate and albumin, on the cefazolin PK in this clinical setting. Another limitation is that our dataset only included total cefazolin concentrations. The unbound concentrations were not measured. This limitation hindered our ability to fully explore the cefazolin maternal protection. Instead, we postulated a 
(a)

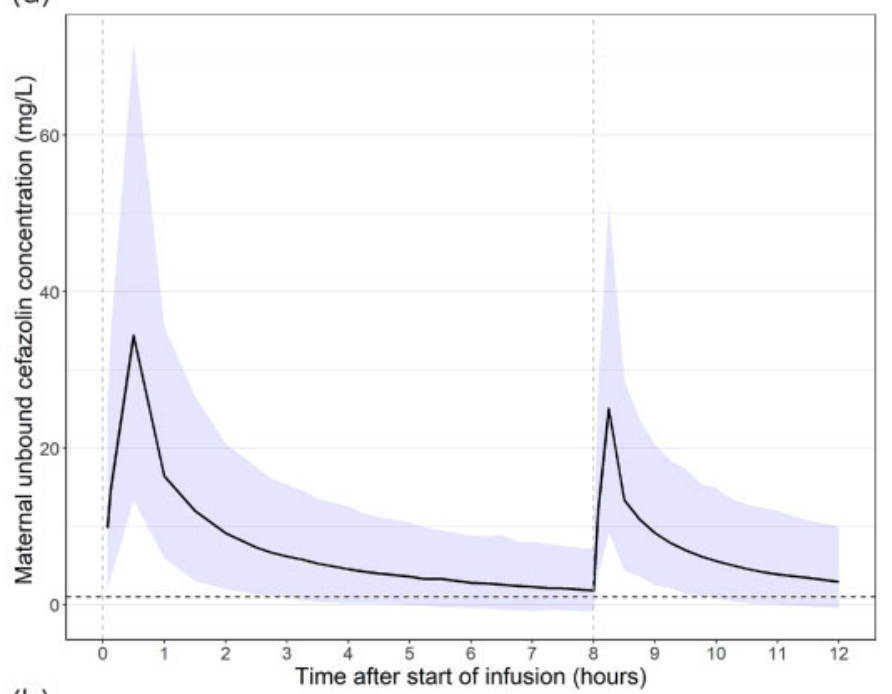

(b)

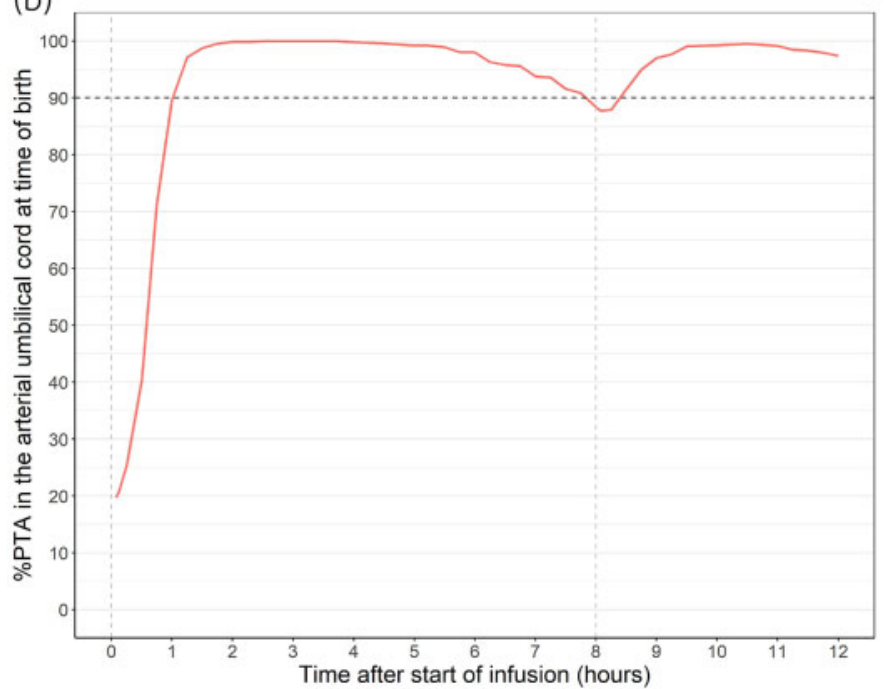

Figure 5. (a) Maternal unbound cefazolin concentrations versus time after the start of the infusion. The black line represents the median of 1000 simulations. The shaded area indicates the $95 \%$ prediction interval of the median. The horizontal broken line indicates the $1 \mathrm{mg} / \mathrm{L}$ therapeutic target. (b) Neonatal PTA at birth versus time since the start of infusion. The horizontal broken line indicates $90 \%$ PTA. This figure appears in colour in the online version of JAC and in black and white in the print version of JAC.

cefazolin protein-bound fraction of $75 \%$ in the maternal blood as previously published. ${ }^{9,10}$

To conclude, we showed that the standard pre-delivery maternal cefazolin dosing provided adequate maternal and neonatal antimicrobial protection against GBS in our patient cohort up to the time of delivery. In addition, we developed a popPK model to describe the cefazolin PK in women during labour with subsequent vaginal delivery. This popPK model was used for simulations that showed that standard pre-delivery cefazolin dosing of a $2 \mathrm{~g}$ infusion at the start and a $1 \mathrm{~g}$ infusion at the eighth hour provides adequate unbound cefazolin concentrations during the entire $12 \mathrm{~h}$ time window of the simulations.

\section{Acknowledgements}

The current work was presented at the 2021 virtual meeting of the Population Approach Group of Australia and New Zealand (PAGANZ) (https://www.paganz.org/abstracts/population-pharmacokinetics-of-cefa zolin-in-maternal-and-umbilical-cord-sera-and-target-attainment-in-termneonates/), the virtual Thirty-First European Congress of Clinical Microbiology \& Infectious Diseases (ECCMID) (https://markterfolg.de/ ESCMID/Final_Programme_2021/index_40.html\#page=32) and the 2021 virtual meeting of the Population Approach Group of Europe (PAGE) (www. page-meeting.org/?abstract=9644).

We thank Professor Catherijne Knibbe for kindly sharing the NONMEM model code of the neonatal cefazolin model by De Cock et al. ${ }^{26}$

\section{Funding}

I.S. is funded by the Clinical Research Fund of the University Hospitals Leuven. A.S.'s research activities are supported by the Clinical Research and Education Council of the University Hospitals Leuven. E.D. is a postdoctoral research fellow of the Research Foundation-Flanders (FWO), Belgium (grant number $12 \times 9420 N$ ). The project was supported in part by a grant of the University Hospital Liège (FIRS).

\section{Transparency declarations}

I.S. has received consultancy support and lecture fees from argenx, Pfizer, Merck and Cidara. E.D. has received consultancy fees from argenx and Janssen (all honoraria/fees paid to the University). All other authors: none to declare.

\section{Supplementary data}

Supplementary data, including Figures S1 to S3 and Tables S1 and S2, are available at JAC Online.

\section{References}

1 Nanduri SA, Petit S, Smelser C et al. Epidemiology of invasive early-onset and late-onset group B streptococcal disease in the United States, 2006 to 2015. JAMA Pediatr 2019; 173: 224-33.

2 Pass MA, Gray BM, Dillon HC. Puerperal and perinatal infections with group B streptococci. Am J Obstet Gynecol 1982; 143: 147-52.

3 Colbourn T, Gilbert R. An overview of the natural history of early onset group B streptococcal disease in the UK. Early Hum Dev 2007; 83: 149-56.

4 Verani JR, McGee L, Schrag SJ; Division of Bacterial Diseases, National Center for Immunization and Respiratory Diseases, Centers for Disease Control and Prevention (CDC). Prevention of perinatal group B streptococcal disease-revised guidelines from CDC, 2010. MMWR Recomm Rep 2010; 59: $1-36$.

5 Briody VA, Albright CM, Has P et al. Use of Cefazolin for group B streptococci prophylaxis in women reporting a penicillin allergy without anaphylaxis. Obstet Gynecol 2016; 127: 577-83.

6 Gyssens IC. Preventing postoperative infections: current treatment recommendations. Drugs 1999; 57: 175-85.

7 Decroix MO, Zini R, Chaumeil JC et al. Cefazolin serum protein binding and its inhibition by bilirubin, fatty acids and other drugs. Biochem Pharmacol 1988; 37: 2807-14.

8 Smits A, Kulo A, Verbesselt R et al. Cefazolin plasma protein binding and its covariates in neonates. Eur J Clin Microbiol Infect Dis 2012; 31: 3359-65. 
9 Vella-Brincat JWA, Begg EJ, Kirkpatrick CMJ et al. Protein binding of cefazolin is saturable in vivo both between and within patients. Br J Clin Pharmacol 2007; 63: 753-7.

10 Allegaert K, Van Mieghem T, Verbesselt R et al. Cefazolin plasma protein binding saturability during pregnancy. Methods Find Exp Clin Pharmacol 2009; 31: 25-8.

11 van Hasselt JGC, Allegaert K, van Calsteren K et al. Semiphysiological versus empirical modelling of the population pharmacokinetics of free and total cefazolin during pregnancy. Biomed Res Int 2014; 2014: 897216.

12 de Hoog M, Mouton JW, van den Anker JN. New dosing strategies for antibacterial agents in the neonate. Semin Fetal Neonatal Med 2005; 10: 185-94.

13 Drusano GL. Prevention of resistance: a goal for dose selection for antimicrobial agents. Clin Infect Dis 2003; 36 Suppl 1: \$42-50.

14 Craig WA. Interrelationship between pharmacokinetics and pharmacodynamics in determining dosage regimens for broad-spectrum cephalosporins. Diagn Microbiol Infect Dis 1995; 22: 89-96.

15 Smaill FM, Grivell RM. Antibiotic prophylaxis versus no prophylaxis for preventing infection after cesarean section. Cochrane Database Syst Rev 2014; issue 10: CD007482.

16 Grupper M, Kuti JL, Swank ML et al. Population pharmacokinetics of cefazolin in serum and adipose tissue from overweight and obese women undergoing cesarean delivery. J Clin Pharmacol 2017; 57: 712-9.

17 Elkomy MH, Sultan P, Drover DR et al. Pharmacokinetics of prophylactic cefazolin in parturients undergoing cesarean delivery. Antimicrob Agents Chemother 2014; 58: 3504-13.

18 Eley VA, Christensen R, Ryan R et al. Prophylactic cefazolin dosing in women with body mass index $>35 \mathrm{~kg} \cdot \mathrm{m}^{-2}$ undergoing cesarean delivery: a pharmacokinetic study of plasma and interstitial fluid. Anesth Analg 2020; 131: 199-207.
19 Muller AE, Dörr PJ, Mouton JW et al. The influence of labour on the pharmacokinetics of intravenously administered amoxicillin in pregnant women. Br J Clin Pharmacol 2008; 66: 866-74.

20 Kunicki PK, Waś J. Simple HPLC method for cefazolin determination in human serum - validation and stability testing. J Chromatogr B Analyt Technol Biomed Life Sci 2012; 911: 133-9.

21 Guilhaumou R, Benaboud S, Bennis Y et al. Optimization of the treatment with $\beta$-lactam antibiotics in critically ill patients - guidelines from the French Society of Pharmacology and Therapeutics (Société Française de Pharmacologie et Thérapeutique-SFPT) and the French Society of Anaesthesia and Intensive Care Medicine (Société Française d'Anesthésie et Réanimation-SFAR). Crit Care 2019; 23: 104.

22 Mouton JW, Muller AE, Canton R et al. MIC-based dose adjustment: facts and fables. J Antimicrob Chemother 2018; 73: 564-8.

23 MIC EUCAST. https://mic.eucast.org/search/show-registration/12248.

24 Wickham H, François R, Henry L et al. dplyr: A Grammar of Data Manipulation. R Package Version 1.0.5. 2021. https://CRAN.R-project.org/pack age $=$ dplyr.

25 Wickham H. ggplot2: Elegant Graphics for Data Analysis. Springer-Verlag New York, 2016.

26 De Cock RFW, Smits A, Allegaert $\mathrm{K}$ et al. Population pharmacokinetic modelling of total and unbound cefazolin plasma concentrations as a guide for dosing in preterm and term neonates. J Antimicrob Chemother 2014; 69: 1330-8.

27 Allegaert K, van Mieghem T, Verbesselt R et al. Cefazolin pharmacokinetics in maternal plasma and amniotic fluid during pregnancy. Am J Obstet Gynecol 2009; 200: 170.e1-7.

28 Muller AE, Oostvogel PM, DeJongh J et al. Pharmacokinetics of amoxicillin in maternal, umbilical cord, and neonatal sera. Antimicrob Agents Chemother 2009; 53: 1574-80. 